Response to Commentaries on Pragmatic Case Studies and Evidence-Based Treatment: Research and Clinical Applications of a Computerized Outcomes Management System

\title{
Practice-Based Evidence and Evidence-Based Practice: The Evidence for Outcomes Management
}

\section{GRANT GRISSOM $^{\mathrm{a}, \mathrm{c}} \&$ JOHN S. LYONS ${ }^{\mathrm{b}}$}

\author{
${ }^{a}$ President and CEO, Polaris Health Directions, Inc. \\ ${ }^{\mathrm{b}}$ Feinberg School of Medicine, Northwestern University \\ ${ }^{\mathrm{c}}$ Correspondence concerning this article should be addressed to Grant Grissom, Polaris Health Directions, \\ 446 Lincoln Highway, Fairless Hills, PA 19030 \\ Email: grant@ polarishealth.com
}

\begin{abstract}
In response to our description of a computer-assisted outcomes management tool for outpatient psychotherapy, Stickle (2006) and Lueger (2006) offer informed and provocative commentaries about both the conceptual and practical aspects of the Polaris-MH system. While we share their enthusiasm for the importance of outcomes data and agree with many of their comments, we differ on some points. In the end, empirical evidence in support of outcomes management generally, and the Polaris-MH specifically, should carry the day.
\end{abstract}

Keywords: outcomes management; computerized assessment; mental health treatment; case studies; expected treatment response (ETR)

\section{RESPONSE TO STICKLE}

We appreciate the thoughtful and informed commentaries provided by Stickle (2006) and Lueger (2006). Responding first to Stickle's comments, we agree that treatment-focused research, or as it is sometimes called, "practice-based evidence," is an important approach to improving psychotherapy relative to the implementation of empirically supported treatments. We share his excitement with the potential of these approaches. We do, however, have a different perspective on three issues that Stickle raised in his commentary.

\section{Data for Non-Patient Populations.}

The first issue is the lack of Polaris MH data for non-patient populations, which Stickle links to assignment of individual patients to functional versus impaired ranges of the Behavioral Health Status (BHS) measure. In our paper we describe the similarity between the Polaris-MH BHS scale and its predecessor, the Mental Health Index (MHI; Grissom \& Lyons, 2006) in 
The Evidence for Outcomes Management

G. Grissom \& J.S. Lyons

Pragmatic Case Studies in Psychotherapy, http://pcsp.libraries.rutgers.edu

Volume 2, Module 3, Article 4, pp. 1-6, 08-18-06 [copyright by authors]

construction and measurement domains. We suggest that the "normal range" of the MHI, derived from both patient and non-patient samples using the method suggested by Jacobson and Truax (1991), is sufficient to meet this need. Stickle's core concern is that, in the absence of BHS data for non-patients, the Truax and Jacobson analysis cannot be carried out directly for the BHS. The functional/normal ranges for the two measures will differ to the extent that the correlation between the two measures is less than unity.

We considered this issue and decided against collecting BHS data from non-patient samples. We feel that the value of specifying functional/impaired ranges with precision does not warrant the associated costs, for two reasons. First, the relationship between the BHS and MHI scores is much stronger than would be evident from the information presented in the paper. More than half of the items that comprise these scales are items that assess symptom severity, and these are identical for the MHI and BHS except that the MHI items are derived from DSM III-R, while the BHS contains the corresponding symptoms from DSM IV. The two remaining domains, subjective well-being and functional disability, share several key items as well. Second, we believe that the specification of a "normal" range is unnecessary to the most meaningful measure of treatment effectiveness, comparison of the patient's progress with the Expected Treatment Response (ETR) curve. We acknowledge that there is value from both clinical and research perspectives to determining whether a patient's condition is "normal." But undue emphasis upon this range can be clinically counterproductive, since "normal" is not a realistic expectation for some patients. And, achieving membership in "the normal range" is not justification for terminating therapy with others. The more important clinical question is: "Is the patient making the progress that would be expected?" given characteristics such as initial clinical severity, treatment history, strengths and so forth. We suggest that the best operational definition of treatment success is progress that equals or exceeds a severity-adjusted expectation (i.e., the ETR), and that this definition does not require specification of a "normal" range.

\section{Patient Characteristics as Predictors of Outcome.}

The second issue raised by Stickle relates to the value of patient characteristics as predictors of outcome. He references Project MATCH (Project MATCH Research Group, 1997) as evidence that patient characteristics may not contribute meaningfully to the prediction of outcome. In our view, the findings of Project MATCH could as readily be interpreted to suggest that, at least for persons with alcohol-related disorders, the treatment model doesn't contribute meaningfully to prediction of outcome. The findings of Project MATCH are entirely consistent with the "Dodo Bird" verdict formerly reserved for mental health treatments: "All have won, and all must have prizes!” (e.g., Wampold, Mondin, Moody, Stich, Benson, Ahn, 1997).

Perhaps more importantly, in our work in the field, we have found that the large majority of therapists believe that patient characteristics have an important bearing upon outcome. Our research data also support this view. We have found that the initial severity of the patient's condition (i.e., the BHS score, a composite of subjective well-being, symptom severity, and functional disability) is an excellent predictor of treatment response, accounting for over $20 \%$ of 
The Evidence for Outcomes Management

G. Grissom \& J.S. Lyons

Pragmatic Case Studies in Psychotherapy, http://pcsp.libraries.rutgers.edu

Volume 2, Module 3, Article 4, pp. 1-6, 08-18-06 [copyright by authors]

outcome variance. Prediction is further improved by measures of treatment expectation, motivation, strengths, and history. As data on the therapeutic Bond/Alliance becomes available, we expect that this will further improve prediction. It is our intention to incorporate whenever feasible additional variables that may improve prediction of ETR, such as therapist assessment of the patient's condition and prognosis, and type of treatment.

\section{Assessing Durability of Effects}

The third issue raised by Stickle is the desirability of assessing durability of effects. We agree that this issue is important in evaluating the effectiveness of treatment. It is rarely done outside of research settings due to the cost of contacting people months after treatment has ended. Our Polaris group has recently concluded development of a web-based version of Polaris$\mathrm{MH}$ that will greatly improve its the feasibility while reducing the cost of post-treatment patient assessments. Interested researchers should contact the first author (Grissom) at Polaris. We do think there is some danger in thinking of treatment as a permanent "cure" rather than a possibly time-limited "fix" that will require additional work in the future. Thus we are not sure that treatment effects that are time-limited are necessarily an indictment of the treatment, but that is a complicated issue for discussion in other venues.

Finally, we share Stickle's view that Polaris-MH holds promise for the refinement of empirically supported treatments. The example that he provides is very useful in suggesting how this might be accomplished. Of course, we also hope that the use of clinical status data compared to expected patterns of response will be of value to therapists who choose not to employ an empirically supported approach. For example, an ETR derived from practitioners using a supported treatment could be employed by a therapist to document the claim that her method is equivalent or superior to that of an empirically supported treatment. Every deviation from a prescribed method constitutes an experiment from which learning can occur, if appropriate data are collected. We hope that Polaris-H will help researchers to better understand the active ingredients in effective treatment, and help clinicians to focus squarely upon the question first proposed by Polaris co-founder Kenneth Howard as of primary importance to quality care: "Is this treatment working for this individual?"

\section{RESPONSE TO LUEGER}

Lueger's commentary provides an accurate description of Polaris-MH features in relation to other systems, and it draws several important inferences regarding the system's potential for improving both research and clinical practice. We share his excitement regarding the potential of systems like Polaris-MH to improve the quality of care, and we agree with the directions he suggests for further development. Given that context, we would like to elaborate upon some aspects of the evolving Polaris-MH system and describe steps that are planned for future enhancement. 


\section{The Phase Model}

Lueger contrasts the development of Polaris MH ("Theoretically-Informed") with that of the OQ45 ("Criterion-Informed"). The choice of Phase Model theory as a basis for the PolarisMH's construction reflects research, clinical, and care management considerations. As noted in our initial paper (Grissom \& Lyons, 2006), the research foundation for phase theory is strong. Clinically, the phases are congruent with the experience and expectations of both outpatient therapists and patients. Clinicians of every major therapeutic school acknowledge improvement in subjective well-being, symptoms, and functioning to be the core goals of treatment, though they differ in their emphases and methods for achieving these outcomes. These domains make sense to patients as well, because they correspond to the familiar experience of medical illness: a sick person feels lousy, experiences symptoms, and these then adversely impact life functioning. Phase theory provides strong face validity, one of the key factors in accurate assessment. The sequence of change provides care managers with useful "flags." For example, the subjective well-being score usually improves within the first 2-4 sessions, indicating remoralization. If the Polaris-MH well-being score does not improve within that time, the case might be slated for closer review and management.

\section{Scale Sensitivity}

Lueger clearly describes the issue of the relationship between a scale's reliability and sensitivity to change, and he correctly notes that the Symptom scale has greater sensitivity than the composite Behavioral Health Status (BHS) scale. In clinical practice therapists are encouraged to focus more upon the pattern or trend in scores, rather than any one score at two points in time. They are further encouraged to review the reports with patients by "drilling down" from the more global BHS score to its three subscales; and within each of the Symptom and Functioning scales, to their respective subscales, and finally to item level data. Therapists are discouraged from attaching significance to minor variations from one assessment to another on any scale. The question of whether a positive trend on a score "really" reflects clinical improvement is often a fruitful focus during the session.

\section{Feedback to Therapists}

The work of Michael Lambert's group (e.g., Lambert, Harmon, Slade, Whipple, \& Hawkins, 2005) in demonstrating the effect of feedback upon treatment outcomes is among the most important advances in the field of outcomes management. We believe that Polaris-MH can build upon this foundation by incorporating measures of patient strengths and other characteristics that we have found to be related to outcome. Per Lueger's comment, we look forward to evaluating the impact of feedback to the therapist of patient progress in relation to expectation following adjustment for patient characteristics with this system (i.e., to the patient's ETR). We have no reason to believe that we will not replicating the findings of Lambert's group. 


\section{Predicting Outcome}

The potential importance of outcomes management is clearly illustrated by the power of predictive systems to impact clinical decisions. Lueger's distinction between descriptive and predictive feedback is useful, but should not be interpreted to mean that comparison of a patient's progress to an expected treatment response (ETR) curve carries no information relating to treatment success or failure. There are patients for whom the curve suggests that "no improvement" should be expected, given the nature of the treatment (e.g., individual or group therapy, with or without medication) and patient characteristics. When data are sufficient to derive an ETR for different treatments, it may be possible to identify the type of treatment that is most likely to produce a favorable outcome. We expect that the ability of the Polaris-MH system to capture information relating to the therapeutic bond, and to incorporate therapist ratings of the patient's status, progress and prognosis, will represent a notable advance in the prediction of treatment outcome.

In closing, we agree with the recommendations of both Stickle and Lueger regarding the directions of further development for Polaris-MH. To that end, features that facilitate integration of Polaris-MH into routine treatment have been as much a focus of development as scientific foundations and clinical utility. For the research implicit in the commentators' recommendations can only be accomplished through the establishment of a large data base reflecting "real world" treatment, which in turn will be accomplished only if the system proves clinically useful and is easily integrated into routine treatment. We welcome the participation in this work of any researchers who would like to use Polaris-MH for their studies, and will honor the wish of our long time collaborator and friend Ken Howard that all interested researchers have full access to the system. At the end of the day, Polaris-MH and other outcomes management approaches can be justified only by empirical data demonstrating that their use results in improved outcomes.

\section{REFERENCES}

Grissom, G. \& Lyons, J. (2006). Pragmatic case studies and evidence-based treatment: Research and clinical applications of a computerized outcomes management system. Pragmatic Case Studies in Psychotherapy [Online], Vol. 2(3), Article 1. Available: http://hdl.rutgers.edu/1782.1/pcsp journal.

Jacobson, N.S. \& Truax, P. (1991). Clinical significance: A statistical approach to defining meaningful change in psychotherapy research. Journal of Consulting and Clinical Psychology, 59, 12-19.

Lambert, M.J., Harmon, C., Slade, K., Whipple, J.L., \& Hawkins, E.J. (2005). Providing feedback to psychotherapists on their patients' progress: Clinical results and practice suggestions. Journal of Clinical Psychology, 61, 165-174.

Lueger, R.J. (2006). Technology to support the clinical management of psychotherapy cases: Commentary on the Polaris-MH. Pragmatic Case Studies in Psychotherapy [Online], Vol. 2(3), Article 3. Available: http://hdl.rutgers.edu/1782.1/pcsp_journal. 
The Evidence for Outcomes Management

G. Grissom \& J.S. Lyons

Pragmatic Case Studies in Psychotherapy, http://pcsp.libraries.rutgers.edu

Volume 2, Module 3, Article 4, pp. 1-6, 08-18-06 [copyright by authors]

Project Match Research Group. (1997). Matching alcoholism treatments to client heterogeneity: Project MATCH Posttreatment drinking outcomes. Journal of Studies on Alcohol, 58(1), 7-29.

Stickle, T.R. (2006). Empirical evaluation of the utility and effectiveness of clinically flexible application in evidence-based treatments. Pragmatic Case Studies in Psychotherapy [Online], Vol. 2(3), Article 2. Available: http://hdl.rutgers.edu/1782.1/pcsp journal.

Wampold, B.E., Mondin, G.W., Moody, M., Stich, F., Benson, K., Ahn, H. (1997). A metaanalysis of outcome studies comparing bona fide psychotherapies: Empiricially, "all must have prizes." Psychological Bulletin. 122, 203-215. 